\title{
Controlled decompression alleviates early brain injury in rabbit intracranial hypertension model by regulating apoptosis/necroptosis
}

\author{
Can Zhang ${ }^{1} \mathbb{D}$, Yue Wang ${ }^{2} \mathbb{D}$, Junhui Chen ${ }^{3} \mathbb{D}$, Shuo Yang ${ }^{4} \mathbb{D}$, Yuhai Wang ${ }^{5, *}$ \\ 1. MM. Department of Neurosurgery - Wuxi Medical College of Anhui Medical University - 904th Hospital of PLA (101th Hospital of PLA) - Wuxi, and \\ Department of Neurosurgery - The Second People's Hospital of HeFei - Anhui, China. \\ 2. MM. Department of Neurosurgery - Wuxi Medical College of Anhui Medical University - 904th Hospital of PLA (101th Hospital of PLA) - Wuxi, China. \\ 3. MD. Department of Neurosurgery - Wuxi Medical College of Anhui Medical University - 904th Hospital of PLA (101th Hospital of PLA) - Wuxi, and \\ Department of Neurosurgery - Renmin Hospital of Wuhan University - Wuhan, China. \\ 4. MM. Department of Neurosurgery - Wuxi Medical College of Anhui Medical University - 904th Hospital of PLA (101th Hospital of PLA) - Wuxi, China. \\ 5. MD, PhD. Department of Neurosurgery - Wuxi Medical College of Anhui Medical University - 904th Hospital of PLA (101th Hospital of PLA) - Wuxi, China.
}

\begin{abstract}
Purpose: To evaluate the effects of controlled decompression and rapid decompression, explore the potential mechanism, provide the theoretical basis for the clinical application, and explore the new cell death method in intracranial hypertension. Methods: Acute intracranial hypertension was triggered in rabbits by epidural balloon compression. New Zealand white rabbits were randomly put into the sham group, the controlled decompression group, and the rapid decompression group. Brain water content, etc., was used to evaluate early brain injury. Western blotting and double immunofluorescence staining were used to detect necroptosis and apoptosis. Results: Brain edema, neurological dysfunction, and brain injury appeared after traumatic brain injury (TBI). Compared with rapid decompression, brain water content was significantly decreased, neurological scores were improved by controlled decompression treatment. Terminal deoxynucleotidyl transferase dUTP nick end labeling (TUNEL) staining and Nissl staining showed neuron death decreased in the controlled decompression group. Compared with rapid decompression, it was also found that apoptosis-related protein caspase-3/ tumor necrosis factor (TNF)-a was reduced markedly in the brain cortex and serum, and the expression levels of necroptosis-related protein, receptor-interacting protein 1 (RIP1)/receptor-interacting protein 1 (RIP3) reduced significantly in the controlled decompression group. Conclusion: Controlled decompression can effectively reduce neuronal damage and cerebral edema after craniocerebral injury and, thus, protect the brain tissue by alleviating necroptosis and apoptosis.
\end{abstract}

Key words: Decompressive Craniectomy. Intracranial Hypertension. RIP. TBI. Necroptosis. Rabbits.

*Corresponding author: wangyuhai1516@163.com | +86 13771166231

Received: Dec 8, 2020 | Review: Feb 13, 2021 | Accepted: Mar 10, 2021

Conflict of interest: Nothing to declare.

Research performed at the Neurology Clinical Center of Wuxi Medical College, the Wuxi Medical College of Anhui Medical University, Wuxi, Jiangsu, China. 


\section{Introduction}

Severe traumatic brain injury (STBI) is a common condition in neurosurgery. According to the epidemiological statistics, the incidence of sTBI is considerably high, which can account for about $10-20 \%$ of the total number of surgical traumas, and has a higher wartime incidence ${ }^{1,2}$. The increased intracranial pressure (ICP) is one of the main causes of death after STBI ${ }^{3,4}$. The standard decompressive craniotomy is currently the conventional surgery for STBI in neurosurgery, since it rapidly decreases ICP to minimize brain damage ${ }^{5}$. However, a previous study has associated traditional standard decompressive craniotomy with the occurrence of several complications, such as intraoperative acute encephalocele and postoperative cerebral ischemia ${ }^{6}$. A study reported that conventional standard decompressive craniotomy can lead to hyperemia and over perfusion after cerebral ischemia ${ }^{7}$. Therefore, more research is required on how to improve the surgery to reduce the incidence of complications.

Controlled decompression is a form of craniotomy that slowly releases ICP during surgery (not rapidly released with conventional standard decompressive craniotomy). This allows for gradual brain tissue reperfusion, thereby protecting cerebral vascular and neurological function ${ }^{8}$. This new randomized, controlled trial (ChiCTR-TCC-13004002) showed that controlled decompression surgery can significantly improve clinical outcomes and reduce the postoperative complications of STBI ${ }^{9}$.

The primary cause of secondary damage in STBI in neuron death. As a passive process of cell death, necrosis was for a long time considered to be uncontrollable ${ }^{10}$. In 2005, Degterev et al. ${ }^{11}$ found that neurons have a controllable type of programmed necrosis, known as necroptosis. Receptor-interacting protein 1 (RIP1) and receptor-interacting protein 3 (RIP3) can activate the signaling cascade under the stimulus of the death signal and cause cell necrosis. This finding is supported by necrostain-1 (NEC-1), a specific inhibitor of RIP1 ${ }^{12,13}$. Necroptosis is common in ischemia-reperfusion injury and may be an effective mechanism of ischemiareperfusion injury ${ }^{14,15}$.

The objective of this study was to evaluate the effects of controlled decompression and rapid decompression, explore the potential mechanism, and provide the theoretical basis for the clinical application, and explore the new cell death method in intracranial hypertension.

\section{Methods}

\section{Experimental animals}

The Bioethics Committee of the Jiangnan University (JN. No. 20190630R0480810[193]) approved the animal procedures used in this study. Procedures were performed following the National Institutes of Health guidelines for handling and care of laboratory animals.

New Zealand White rabbits (weighing 2.2-2.5 kg, 3 months old) were obtained from the Animal Central of Taihu Hospital (Wuxi, China). They were maintained at a normal level of atmospheric humidity room with a constant temperature $\left(22^{\circ} \mathrm{C}\right)$ and a $12 \mathrm{~h}$ photoperiod for at least 15 days before the experiment. They had free access to food and water. Rabbits without anomalies were selected, fasted for $12 \mathrm{~h}$, and used for the experiment.

\section{Establishing intracranial hypertension model rabbit}

The protocol of intracranial hypertension model rabbit was described as a previous study ${ }^{16}$. After $12 \mathrm{~h}$ of fasting, the rabbits were anesthetized with intramuscular ketamine (50 $\mathrm{mg} \cdot \mathrm{kg}^{-1}$ ), and continuous intravenous infusion of sodium pentobarbital $\left(10 \mathrm{mg} \cdot \mathrm{kg}^{-1} \cdot \mathrm{h}^{-1}\right)$ via the marginal ear vein throughout the remainder of the experiment. The rabbits were placed in a prone position and fixed on the operating bench after being anesthetized. The hair on the head was shaved and the skin on the operation area disinfected. The scalp was then cut open at the center of the head and the skull was dissected. Using a dental drill, bone holes with a diameter of $0.3 \mathrm{~cm}$ were drilled behind the coronal suture and $0.5 \mathrm{~cm}$ on the left and right sides of the sagittal suture. Bone residues were removed carefully to prevent the exposure and damage of dura mater and blood vessels. The ICP express was inserted into brain tissue about $1.0 \mathrm{~cm}$ deep in the left vertical direction. The epidural pressure balloon was placed in the right frontotemporal direction (Fig. $1 \mathrm{a}-\mathrm{C}$ ). The ICP probe was connected to an ICP monitoring device (Codman, Johnson and Johnson Medical Ltd, 82-6635, USA) to observe ICP changes during operation. An epidural compression balloon was connected to an intracranial infusion pump (Merit Basix Touch Inflation Device, IN4130, USA). 

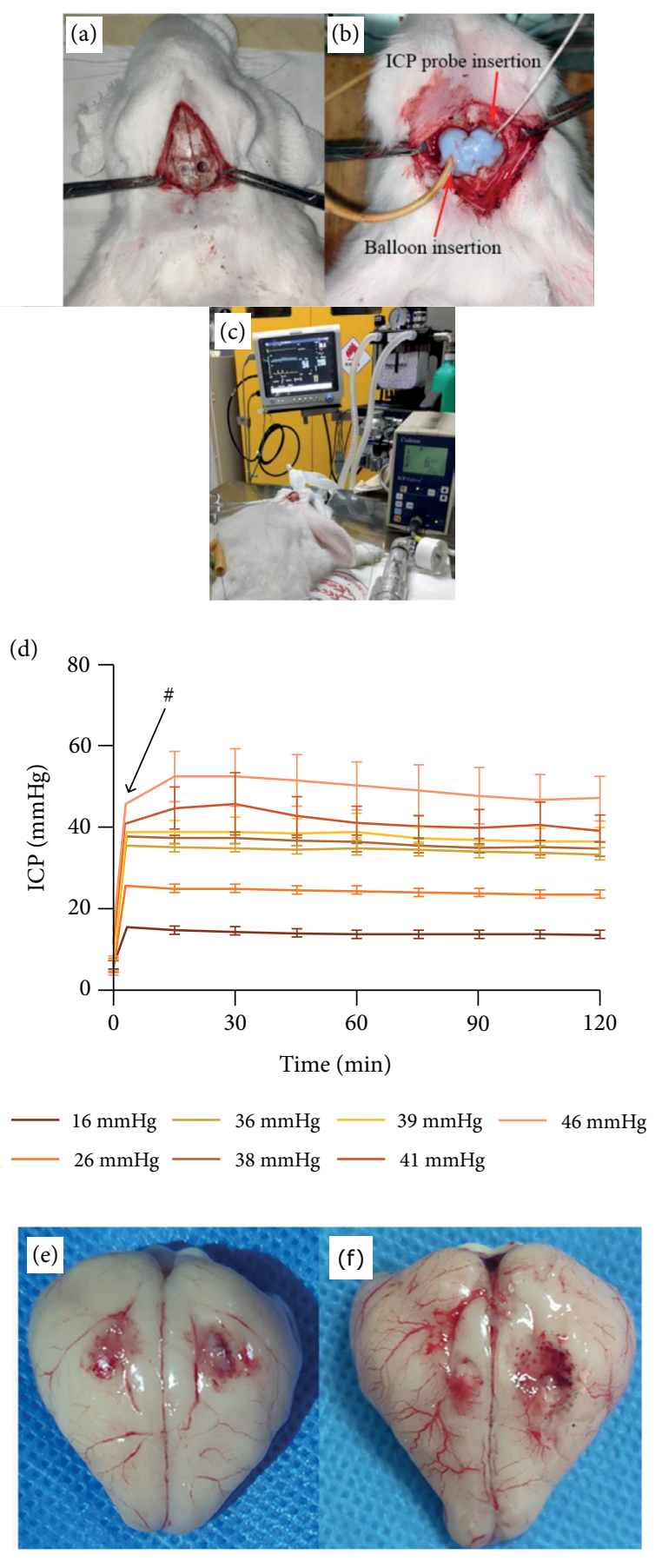

Figure 1 - $(\mathbf{a}-\mathrm{c})$ The process of establishing the model of intracranial hypertension; (d) ICP changes in each group within $120 \mathrm{~min}$. (\# represents that each group has completed the injection of water into the balloon within 3 min); (e) Gross anatomy of the controlled decompression group; (f) Gross anatomy of the rapid decompression group Red arrow indicated damaged area.

\section{Experimental design}

To explore the limit of ICP, $10 \mathrm{mmHg}$ was increased in each group according to the standard ICP $(6 \mathrm{mmHg})$ of New Zealand rabbits. The ICP was increased to the corresponding value 3 min into the experiment. Intracranial pressure, heart rate, respiratory, and mortality values were observed after $120 \mathrm{~min}$. When most of the experimental animals exhibited a sharp ICP fluctuation (greater than or equal to $15 \%$ of the original ICP value) or a significant increase in mortality within $24 \mathrm{~h}$, the ICP threshold was assumed to have been reached. Dynamic monitoring of ICP, the average of the ICP value that reached the ICP limit and the ICP value that did not reach the limit in the previous group were used as the ICP setting value of the next group (take an integer). The ICP value approaching the limit according to the actual situation was divided into 7 groups: $(16,26,36,38,39,41$ and $46 \mathrm{mmHg})$. There were four rabbits in every group.

The animals were divided into three groups ( $n=8$ /group) after the verification of the ICP limit value. The sham group, which did not inject liquid after placing the epidural pressurized balloon and keeps it for $30 \mathrm{~min}$. In the controlled decompression group, after placing the epidural pressurized balloon, the ICP was increased to $38 \mathrm{mmHg}$ within $10 \mathrm{~min}$ and maintained for $30 \mathrm{~min}$. The pressure infusion pump was then used to make the ICP decrease uniformly to a normal ICP level within $30 \mathrm{~min}$. In the rapid decompression group, the treatment was the same as in the controlled decompression group, but the ICP was released much faster than in the controlled decompression group, within $10 \mathrm{~s}$. The balloon and ICP probe were removed, then bone wax and dental resincement (Clearfil SA Cement, RH Dental, Denmark) were used to seal the bone holes and suture the scalp. After recovery from anesthesia, the rabbits were released into the feeding room. All the rabbits were euthanized with $100 \mathrm{mg} \cdot \mathrm{kg}^{-1}$ of intravenous pentobarbital injections after $24 \mathrm{~h}$ of observation in the feeding room. The cerebral cortex under balloon compression (about $5 \mathrm{~mm}^{2}$ ) was removed immediately after brain dissection on ice for western blot detection and Nissl staining. The other portion was used to estimate brain tissue edema.

\section{Neurological scoring}

An independent observer who was blinded to the research recorded neurological scores $24 \mathrm{~h}$ after surgery. A previously modified neurological scoring table (Table 1) was used to assess the neurological function post-operation $^{17,18}$. 
Table 1 - Neurological scoring among the three experimental groups.

\begin{tabular}{lcc} 
Category & Behavior & Score \\
\cline { 2 - 3 } Appetite & Finished meal & 0 \\
\cline { 2 - 3 } & Uneft meal unfinished & 1 \\
\cline { 2 - 3 } Activity & Active, squeaking or standing & 2 \\
\cline { 2 - 3 } & $\begin{array}{c}\text { Lying down, will stand and walk } \\
\text { with some stimulation }\end{array}$ & 1 \\
\hline \multirow{2}{*}{$\begin{array}{c}\text { Almost always lying down } \\
\text { No deficits }\end{array}$} & 2 \\
\cline { 2 - 3 } Deficits & $\begin{array}{c}\text { Unable to walk due to ataxia or } \\
\text { paresis }\end{array}$ & 1 \\
\cline { 2 - 3 } & $\begin{array}{c}\text { Impossible to walk and stand due } \\
\text { to ataxia and paresis }\end{array}$ & 2 \\
\hline
\end{tabular}

\section{Brain water content}

The standard wet-dry method was used to compute the brain water content, as previously reported ${ }^{19,20}$. The entire brain tissue was taken for water content detection immediately after the animals were killed. After dissection, the brain tissue was promptly placed on a precision electronic scale with tinfoil to record the wet weight (M); the brain tissue was then packaged using tinfoil and put into a drying oven at $110^{\circ} \mathrm{C}$ for $24 \mathrm{~h}$. The tissue was weighed to measure the dry weight $(\mathrm{m})$ after restoring the temperature to room temperature. Brain water content $=(\mathrm{M}-\mathrm{m}) / \mathrm{M} \times 100 \%$.

\section{Cytokine tumor necrosis factor (TNF)-a measurements}

TNF-a was measured by ELISA (Thermo Fisher, A356015) according to the manufacturer's instructions.

\section{Nissl staining}

Nissl staining was performed as described in the previous study $^{21}$. Briefly, the cerebral cortex was post-fixed in $4 \%$ paraformaldehyde at $4{ }^{\circ} \mathrm{C}$ overnight, dehydrated and embedded in paraffin, and sectioned $(4 \mu \mathrm{m})$. The paraffin sections were torrefied at $60^{\circ} \mathrm{C}$ for 30 min then dewaxed using xylene. Gradient alcohol dehydration was then followed by slow flushing with running water for $1 \mathrm{~min}$. The sections were then stained with toluidine blue at room temperature for $15 \mathrm{~min}$. After washing slowly with running water, $1 \%$ hydrochloric acid alcohol to differentiate, then washed again by running water. The sections were then counterstained in a saturation lithium carbonate solution for $30 \mathrm{~s}$. Finally, they were dehydrated with alcohol, transparentized by xylene, and sealed with gum.
An Olympus light microscope (Olympus Corporation, Tokyo, Japan) and Image-Pro Plus 6.0 Software (Media Cybernetics, Inc., Rockville, MD, USA) were used to determine the numbers of Nissl bodies in neurons.

\section{Measurement of apoptosis by terminal deoxynucleotidyl transferase dUTP nick end labeling (TUNEL) staining}

Apoptosis in neurons was determined using a standard TUNEL staining method according to the manufacturer's protocol (Roche, Penzberg, Germany).

\section{Double immunofluorescence staining}

The basic methods of double immunofluorescence staining are described in previous studies ${ }^{19,20}$. The necroptosis in the rabbit cerebral cortex was evaluated by RIP3 and neuronal staining specific marker (NeuN) double immunofluorescence staining. Briefly, the rabbit cerebral cortex was fixed in $4 \%$ paraformaldehyde for $24 \mathrm{~h}$ at $4{ }^{\circ} \mathrm{C}$, and a $30 \%$ sucrose solution was used to dehydrate the sample. The samples were then sectioned to a thickness of $10 \mu \mathrm{m}$. Many of the procedures, including Nissl staining, were similar to histologic staining. The primary antibodies, including anti-NeuN polyclonal antibody (1:200, ab128886; Abcam) and anti RIP3 polyclonal antibody (1:500, bs-3551R; Bioss, China), were diluted overnight in PBS at $4{ }^{\circ} \mathrm{C}$. The goat anti-rabbit IgG secondary antibody (1:500; Beyotime, China) was incubated at room temperature for $1 \mathrm{~h}$ after washing and rinsing the sections in PBS. It was incubated and covered with DAPI, rinsed again, and covered with glycerol. Fluorescence microscopy (Leica Microsystems, Germany) was used to observe and count the positive cells.

\section{Western bolt analysis}

Western bolt analysis was used to determine the levels of RIP1, RIP3, and caspase-3 proteins and performed as described previously. The cerebral cortex sample from the left compression area was collected and homogenized to extract protein. Samples were then mixed with sodium dodecyl sulfate-polyacrylamide gel electrophoresis (SDS-PAGE) loading buffer, heated at $100^{\circ} \mathrm{C}$ for $5 \mathrm{~min}$, cooled on ice, and centrifuged for $5 \mathrm{~min}$ to remove the insoluble precipitate. Samples with a maximum sample size of $20 \mu \mathrm{L}$ per pore were separated by $10 \%$ SDS-PAGE and transferred to a nitrocellulose membrane (Bio-Rad, USA). Rabbit antibodies against RIP1 (bs-5805R; Bioss; China), RIP3 (bs-3551R; Bioss; China), caspase-3 (ab44976; Abcam; USA) were probed with the membrane and overnight at $4{ }^{\circ} \mathrm{C}$. HRPconjugated goat anti-mouse IgG secondary antibodies (1:1000; Sigma) were added to the membrane after rinsing with TBST and incubated for $2 \mathrm{~h}$ at $37^{\circ} \mathrm{C}$. Super-GL ECL hypersensitive 
luminous solution was used to detect chemiluminescence, and the X-ray film was exposed. The dried film was eventually photographed with the gel imaging analysis system (GelPro Analyzer software [Media Cybernetics, Inc.]) after the development and fixing processing.

\section{Statistical analysis}

All the data were presented as the mean \pm standard deviation, and analysis was done in SPSS (version 23; IBM, New York, USA). One-way analysis of variance and least significant difference (LSD) were used for multiple comparison tests. Statistical significance was set at $p<0.05$.

\section{Results}

\section{Mortality and ICP setting}

In this experiment, the mortality of the controlled decompression group, rapid decompression group, and the sham group were $11.1 \%(1 / 9), 20 \%(2 / 10)$, and $0 \%$ $(0 / 8)$. Additionally, the reasons for animals' death after surgery can be seen in Table 2. Besides, in the preexperiment phase, it was observed that when the ICP of the experimental animals rose above $38 \mathrm{mmHg}$ : $58.3 \%$ of the ICP of experimental animals fluctuated sharply; apnea, deep breath, and pulmonary signs, such as apparent wet crackles, occurred in $41.7 \%$ of the experimental animals; $25 \%$ of the experimental animals died within 24 $\mathrm{h}$ after surgery, and $16.7 \%$ of the experimental animals died during surgery. Thus, the limit of ICP was set to 38 $\mathrm{mmHg}$ in the formal experiment (Fig. 1d). To maintain the number of animals in each group, the dead rabbits were replaced during the model establishment process. The compression area of the control and rapid decompression group collapsed more than the compression area of the control group. The red arrow shows the spot hemorrhage. There was no difference on the contralateral side (Fig. 1e).

Table 2 - Cause and number of deaths.

\begin{tabular}{|c|c|c|c|c|c|c|c|}
\hline \multirow{2}{*}{$\begin{array}{l}\text { Group/Cause } \\
\text { of death }\end{array}$} & 16 & 26 & 36 & 46 & 41 & 39 & 38 \\
\hline & \multicolumn{7}{|c|}{$(\mathrm{mmHg})$} \\
\hline $\begin{array}{c}\text { Subdural } \\
\text { hematoma }\end{array}$ & 0 & 0 & 0 & 0 & 0 & 0 & 0 \\
\hline $\begin{array}{c}\text { Epidural } \\
\text { hematoma }\end{array}$ & 0 & 0 & 1 & 2 & 1 & 1 & 1 \\
\hline $\begin{array}{l}\text { Respiratory and } \\
\text { circulatory disorders }\end{array}$ & 0 & 0 & 0 & 3 & 1 & 1 & 0 \\
\hline Unknown reason & 0 & 0 & 0 & 0 & 0 & 1 & 0 \\
\hline $\begin{array}{l}\text { Total number of } \\
\text { deaths }\end{array}$ & 0 & 0 & 1 & 5 & 2 & 3 & 1 \\
\hline
\end{tabular}

\section{Controlled decompression improves neurological scoring and brain water content}

The neurological scoring of animals in the controlled decompression group and the rapid decompression group increased significantly compared with the sham group at $24 \mathrm{~h}$ after surgery ( $p<0.01$; Fig. 2 ; according to ANOVA). Moreover, neurological scoring in the control group decreased significantly compared with the rapid group at $24 \mathrm{~h}$ after surgery ( $\mathrm{p}<0.01$; Fig. $2 \mathrm{a}$; according to ANOVA). The brain water content of the control group and the rapid group were significantly increased (80.5 vs. 79.4, p < 0.05; 81.5 vs. 79.4, $\mathrm{p}<0.01$ ) compared to the Sham group at $24 \mathrm{~h}$ postoperation. The brain water content in the controlled decompression group decreased significantly compared with the rapid decompression group (80.5 vs. 81.5, $p<0.05$ ) (Fig. 2b).

\section{Controlled decompression alleviates neuron apoptosis}

The image of Nissl staining shows that cortical nerve cells in the rapid decompression group and the controlled decompression group are sparsely distributed and disorganized compared with the sham group. Compared with the controlled decompression group, the rapid decompression group had visible vacuoles around the cell body, and the staining became lighter. The number of positive cells of Nissl body in the controlled decompression group and the rapid decompression group increased significantly compared with the sham group at $24 \mathrm{~h}$ after surgery $(\mathrm{p}<0.01)$. However, there was no significant difference in the number of positive cells of Nissl body between the controlled decompression group and the rapid decompression group ( $p>0.05$ ) (Fig. $2 c)$. Western blot (WB) was used to detect caspase-3 protein expression levels in the cortex. To observe the apoptosis at $24 \mathrm{~h}$ after surgery, the rapid group induced a notable increase of caspase- 3 expression levels in the cortex compared with the sham group, whereas the level of caspase-3 was substantially decreased in the control group. Furthermore, compared with the rapid group, the control group decreased significantly (Fig. 3a). It was also found that the control group decreased the TNF-a expression levels significantly in the serum (Fig. 3c) and cerebral cortex (Fig. 3b). The TUNEL staining showed that hippocampus neuron apoptosis in the rapid decompression group and the controlled decompression group were increased compared with the sham after surgery. Besides, in the controlled decompression group, the neurons apoptosis decreased compared with the rapid decompression group (Fig. 4). 
(a)

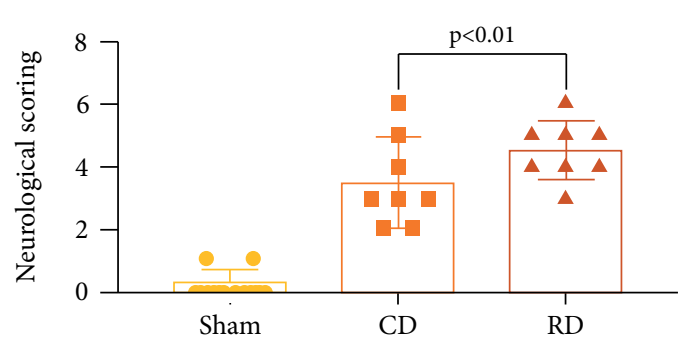

(b)

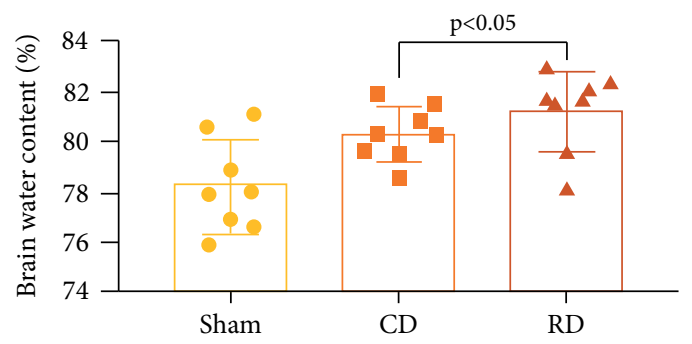

(c)
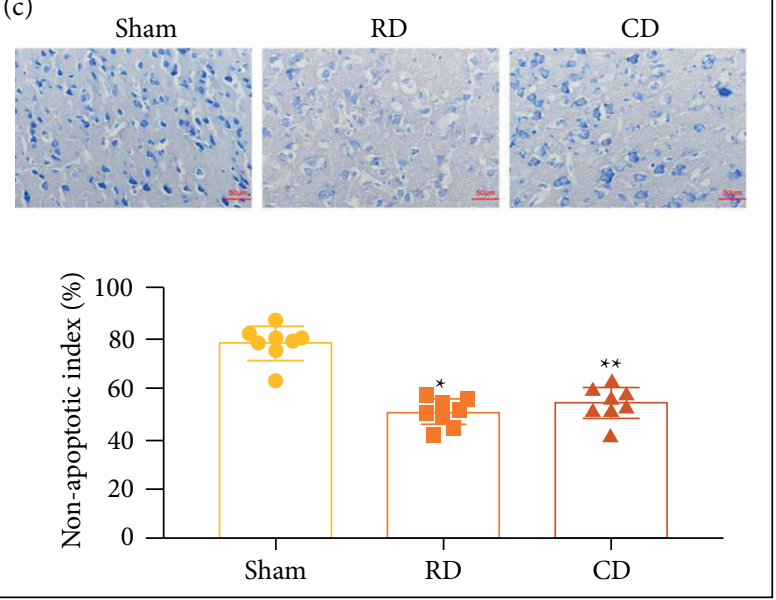

Figure 2 - Controlled decompression decreases the neurological scoring and brain water content after surgery. (a) The results indicated that neurological scoring was significantly lower in the sham group compared with the rapid group and the control group. The sham group $v s$. the control group and the rapid group, $p<0.01$; the control group vs. the rapid group, $p<0.01$. (b) Graphs showing the brain water content of the three groups. The sham group vs. the control group and the rapid group, $\mathrm{p}<0.05$ and $\mathrm{p}<0.01$; the control group vs. the rapid group, $p<0.05$ ( $n=8$, mean \pm standard deviation (SD), one-way analysis of variance). (c) Nissl staining of cortex following different surgical methods. The neurons were sparsely distributed and disorderly arranged in the compression area of cortex in the rapid group and the control group compared with the sham group, and significant variance in several positive cells of Nissl body was observed in the rapid group and the control group, the rapid group are obvious vacuoles around the cells, and the staining of Nissl body became shallow. For statistical analysis, no significant statistical difference in the number of Nissl body-positive cells (number/100 $\mathrm{mm}^{2}$ ) in the control group compared with the rapid group; ${ }^{*} p<0.01$ vs. sham group, $* * p<0.05 v s$. sham group $(n=8)$.

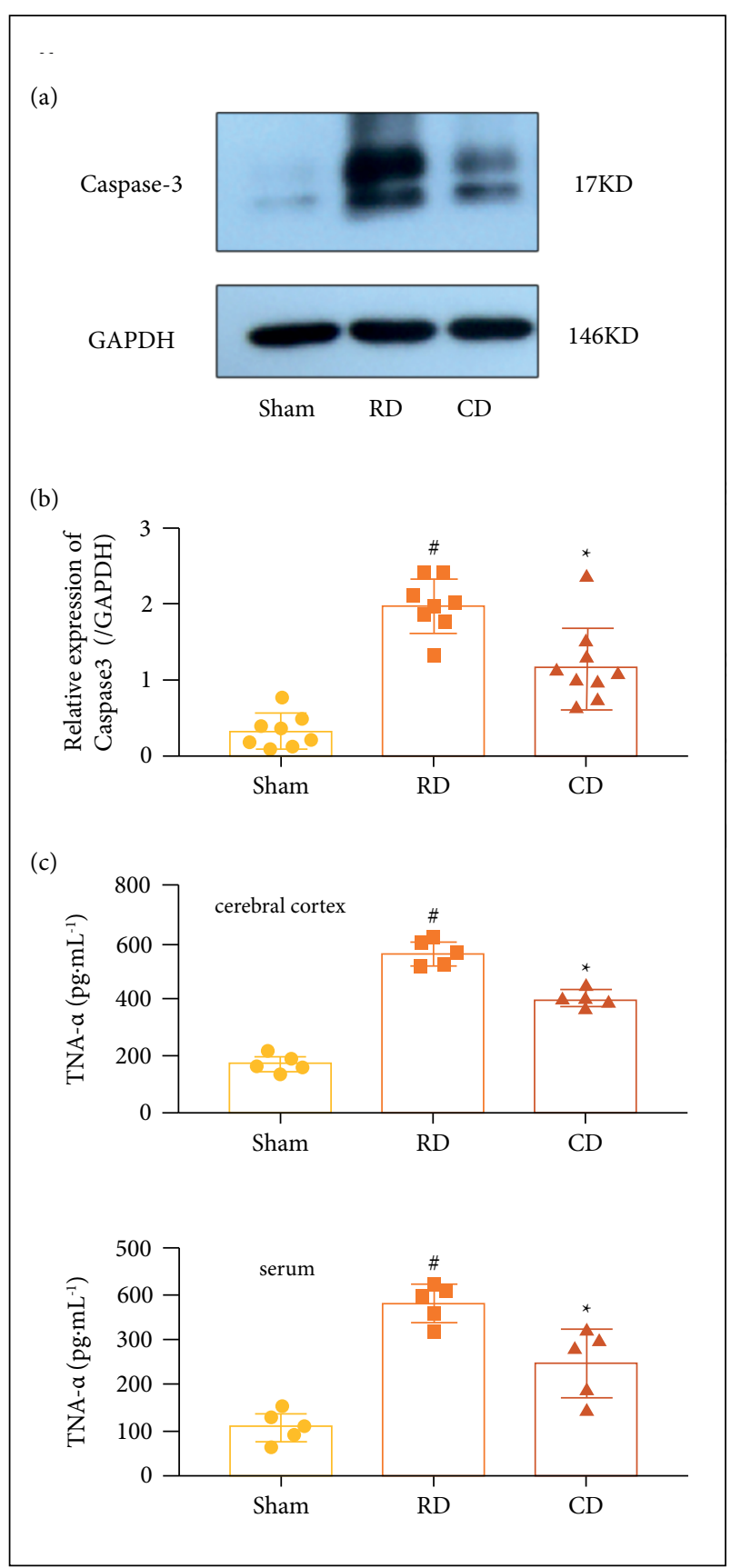

Figure 3 - Controlled decompression decreases the apoptosis-related protein expression in the cortex and serum. (a) Representative western blot of caspase-3 protein expression. Caspase-3 protein expression in the Sham group was significantly lower than the control group and the rapid group ( $n=8 /$ group). Caspase- 3 expression levels were significantly decreased in the control group. (b) The control group decrease the levels of TNF-a in the cerebral cortex by ELISA $(n=5)$. (c) The control group decrease the levels of TNF-a in the serum by ELISA $(n=5) * p<0.01$ vs. sham group, $\# p<0.01$ vs. sham group and rapid decompression (RD) group. 


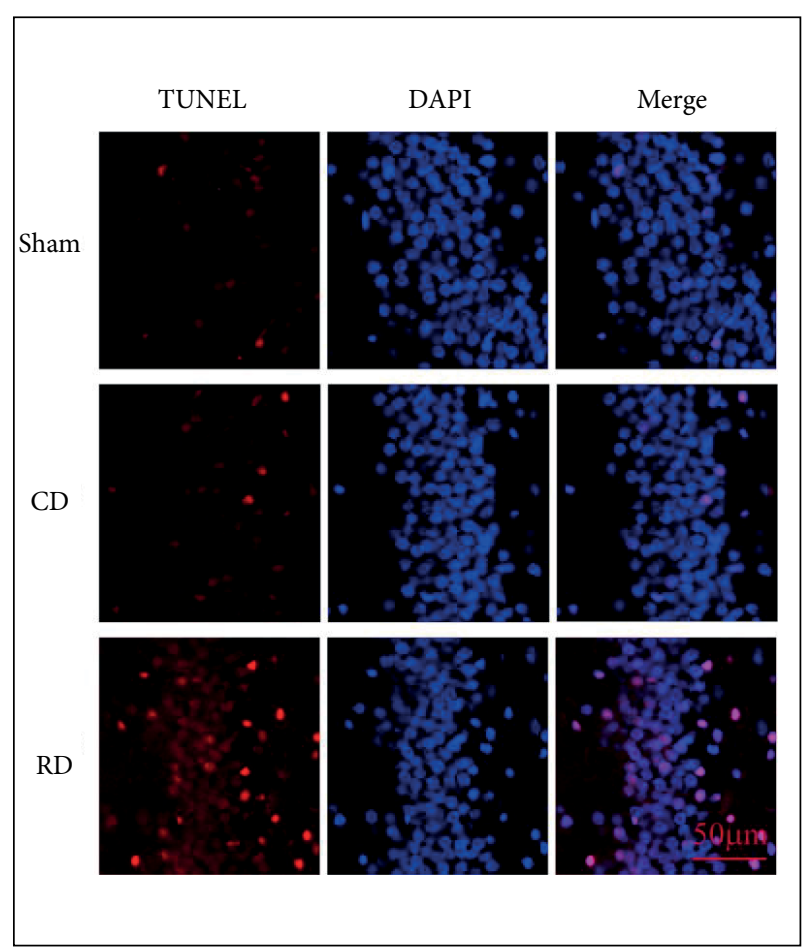

Figure 4 - Controlled decompression decreases the neuron apoptosis by TUNEL staining in the hippocampus. It showed that controlled decompression can inhibit neuronal apoptosis compared with the rapid group; apoptosis neuron is enhanced after intracranial hypertension and decreased after controlled decompression.

\section{Controlled decompression alleviates neuron necroptosis}

In this study, WB was used to assess RIP1 and RIP3 expression levels in the cerebral cortex $24 \mathrm{~h}$ after surgery. The expression of RIP1 and RIP3 in the controlled decompression group (RIP1: $p<0.05$; RIP3: $p<0.01$ ) and the rapid decompression group (RIP1: $p<0.01$; RIP3: $p<0.01$ ) significantly increased compared to the expression levels of RIP1 and RIP3 in the sham group. However, the expression levels of RIP1 ( $p<0.01)$ and RIP3 ( $p<0.05)$ in the controlled decompression group were significantly lower than in the rapid decompression group (Fig. 5). After intracranial hypertension induction, double immunofluorescent staining showed that RIP3-positive cells were widespread. Besides, more RIP3 positive neurons were observed in the rapid decompression group compared with the controlled decompression treatment group (Fig. 6). (a)
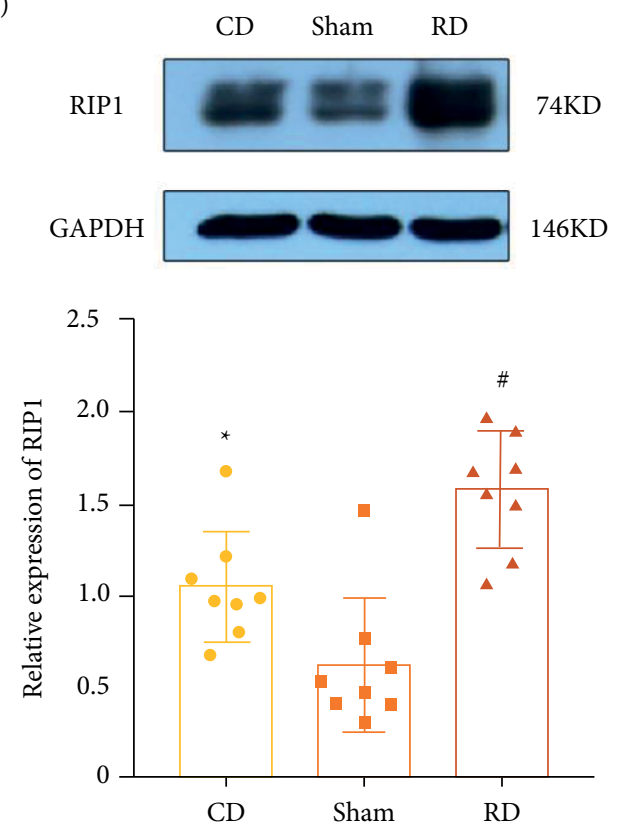

(b)
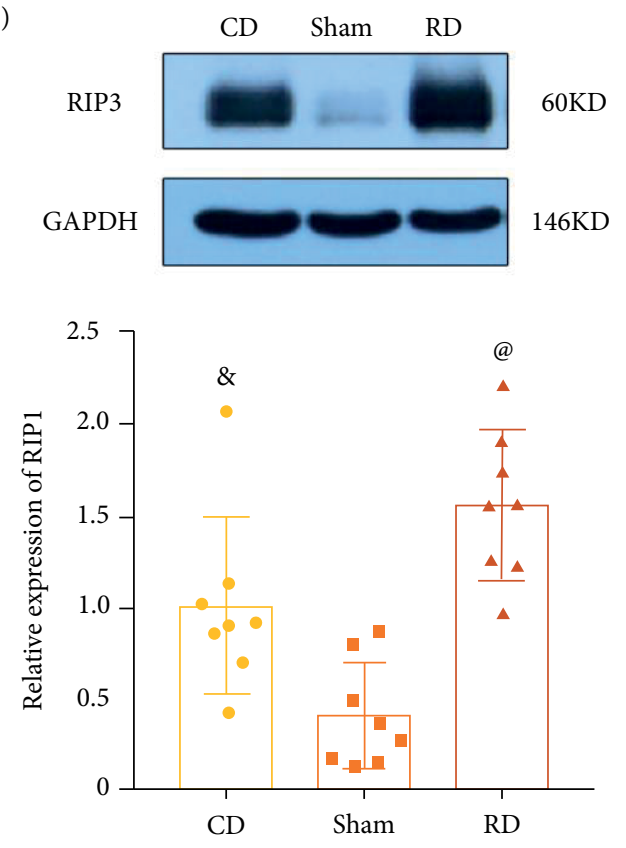

Figure 5 - Controlled decompression decreases the expression of RIP1 and RIP3 by western blot (WB). (a) Representative WB of RIP1 protein expression. ${ }^{*} p<0.05$ vs. sham group, $\# p<0.01$ vs. sham group and Controlled decompression (CD) group. (b) Representative WB of RIP3 protein expression. \& $<<0.01$ vs. sham group, @p $<0.01$ vs. sham group and CD group. $(n=8)$. 


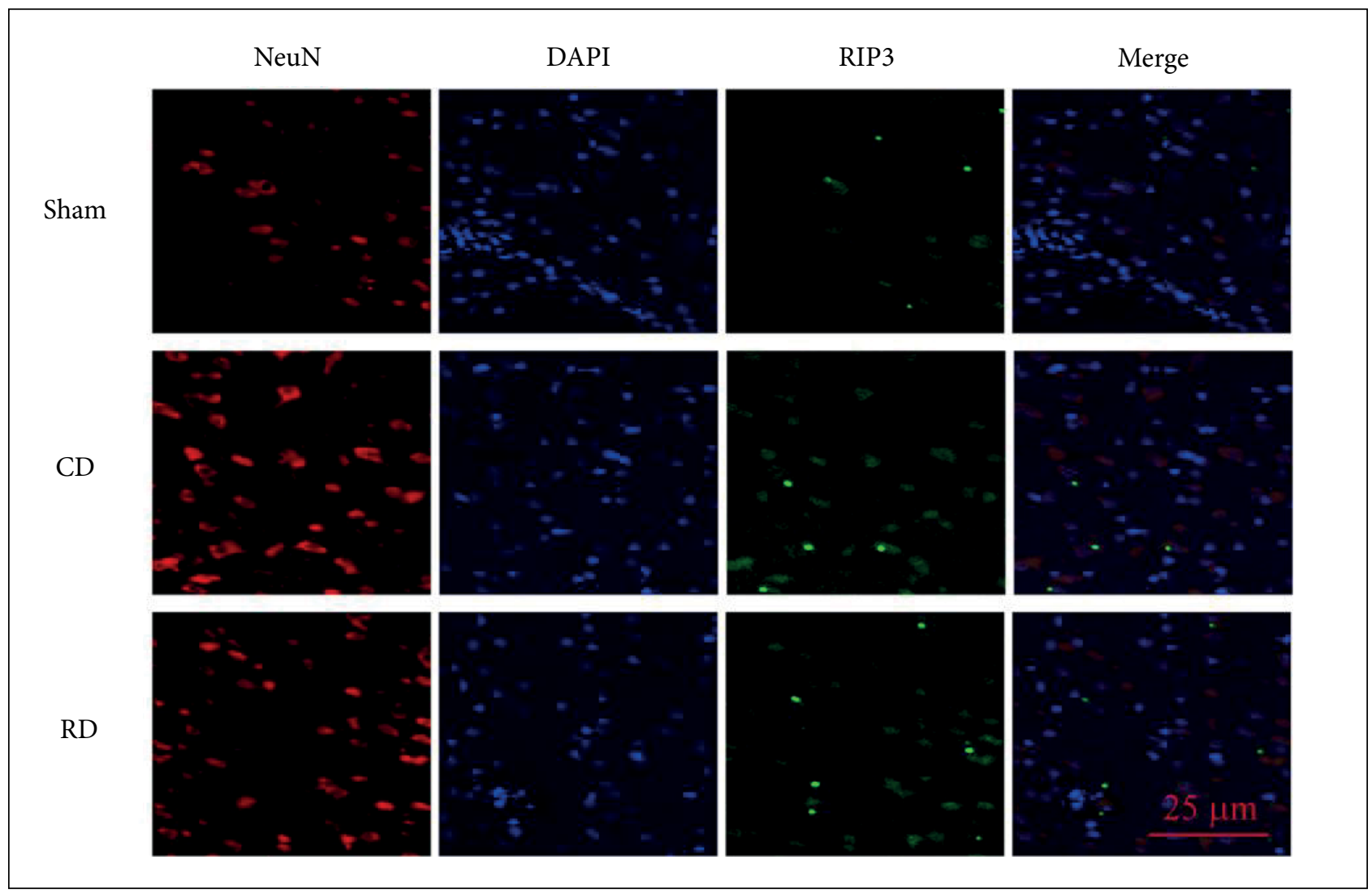

Figure 6 - Controlled decompression decreases the expression levels of RIP3. Immunofluorescent staining of RIP3/NeuN/DAPI in the cerebral cortex indicated that controlled decompression can inhibit the expression of RIP3; RIP3 immunoreactivity is enhanced after intracranial hypertension and decreased after controlled decompression.

\section{Discussion}

In this study, the mortality rate, brain water content, and neurological scores were assessed. Nissl staining was also used to evaluate neuron death in three surgical modes. Western blotting and double immunofluorescence staining were eventually used to determine the protein levels of RIP1, RIP3, and caspase-3. These experimental results undoubtedly confirmed that controlled decompression can effectively reduce ICP and inhibit the necroptosis pathway as well. Besides, previous clinical trials also improved surgical patient prognosis and decreased the incidences of postoperative complications ${ }^{8,9}$.

Severe traumatic brain injury remains the primary cause of mortality in adults worldwide and is currently one of the biggest public health concerns. Moreover, sTBI complications are still common. The best approach to help these patients could be the application of decompressive craniectomy $^{22}$ with dural augmentation ${ }^{23}$. It has the advantages of full decompression, providing more and larger intracranial buffer space for brain tissue under high pressure, reducing ICP, rebuilding cerebral blood flow perfusion, and controlling secondary brain damage. In the surgical procedure, rapid decompression and rapid removal of hematoma are usually adopted to reduce ICP, which, in turn, increases cerebral blood perfusion and decreases secondary brain injury as well. Although some studies have shown that DC can substantially reduce ICP in patients with severe brain injury, the incidence of complications, including cerebral infarction, remains high. Similarly, morbidity and mortality are still as high as $50-70 \%{ }^{6,23}$. Thus, reducing the incidence of intraoperative and postoperative complications remains a major challenge. Mcleod stated that some of the complications might be attributed to the rapid removal of bone flaps and hematoma during decompressive craniectomy ${ }^{7}$. The rapid decrease of ICP in contralateral brain tissue, and the absence of the impact of pressure tamponade, results in a rapid hemorrhage of ruptured dural arteries or broken plate barriers, and can even cause hemostatic dural rupture bleeding? 
Based on the research of Su et al., compressed cerebral blood vessels rapidly expand and become congested after rapid decompression, leading to excessive perfusion of cerebral blood vessels ${ }^{24}$. However, if the perfusion of cerebral blood vessels exceeds a certain threshold, it can damage the blood-brain barrier, increase cerebral blood vessel permeability, and cause excessive plasma components leakage, thus aggravating vasogenic cerebral edema and even causing acute brain swelling ${ }^{24}$. Jiang et al. also confirmed that low-pressure perfusion can enhance the recovery of spinal cord function after ischemia and alleviate spinal cord injury caused by reperfusion in rats ${ }^{25}$. Tamaki et al. also stated that a rapid reduction in ICP can lead to serious adverse changes in hemodynamics ${ }^{26}$. Therefore, to achieve a stable cerebral perfusion pressure, intracranial hematoma should be gradually removed and ICP gradually reduced ${ }^{26}$. Based on the previous studies and clinical experience, Wang et al. first reported a modern treatment mode-controlled decompression, which could control the amount of cerebral perfusion, and transform the one-time perfusion into gradually controlled perfusion ${ }^{8}$. It has since achieved excellent clinical effect ${ }^{8}$. Controlled decompression technology can protect nerve cells and function by reducing ischemia-reperfusion damage and maintaining vascular regulation. This clinical study also demonstrated that the 6-months outcomes, the incidence rates of intraoperative acute brain swelling, and delayed intracranial hematoma were significantly better in STBI patients who underwent controlled decompression than in those who underwent rapid decompression ${ }^{9}$. Rapid craniotomy (opening the skull and dura quickly, without controlled ICP release) causes large amounts of arterial blood to pour quickly into the brain tissue, but without appropriate venous outflow, then lead to brain edema and brain swelling. The exact mechanism of its action is yet to be established. The pathophysiological process of nerve cell damage after traumatic brain injury (TBI), such as apoptosis and necrosis of nerve cells, should be studied. This will help in exploring the potential molecular mechanism of decompression technology and reduce the incidence of postoperative complications and protect brain tissue ${ }^{27}$.

Necroptosis is a new type of cell death with typical necrotic morphology regulated by signal molecules, including RIP1 and RIP3 in cells ${ }^{28}$. Receptor-interacting protein 3 can phosphorylate RIP1, which can form a stable necrosome complex, activate its downstream necrosis kinase, and eventually result in necroptosis, which can be prevented by a small molecule inhibitor NEC- $1^{13,29}$. In recent years, a previous study has proven that necroptosis exists in brain tissue neurons, and is correlated with the neurotoxic effect of 24S-Hydroxycholesterol (24S-OHC) on cortical neurons ${ }^{30}$. Liu reported that intraventricular NEC-1 injection can greatly alleviate brain edema and neuron death in mice after craniocerebral injury ${ }^{31}$. Hypoxia in brain tissue can cause Receptor-interacting protein kinases 3 (RIPK3) upregulation. Receptor-interacting protein 1 and RIP3 upregulation aggravate the death of hippocampal neurons. Also, Previous study have shown that induces the processing of cerebral ischemia-reperfusion. Yang et al. had demonstrated that, after cerebral ischemia, the expression levels of RIP3 and mixed-lineage kinase domain-like protein (MLKL) in neurons and astrocytes increased and the area of cerebral infarction caused by ischemia was significantly reduced after RIP3 or MLKL gene knockout ${ }^{32}$. Previous studies have established that necroptosis plays a key role in the pathological process of brain tissue injury, which can, to some degree, intensify brain tissue injury. Therefore, it was speculated that controlled decompression technology can, to a certain extent, block necroptosis, reduce neuron death, improve ischemia-reperfusion injury, reduce the infarct area of brain tissue, and reduce brain edema.

Tumor necrosis factor (TNF)-a is implicated in both pathways of apoptosis and necroptosis and also a key cytokine eliciting the early inflammatory cascades in ischemia-reperfusion injuries. In the present study, it was also found that the expression levels of TNF-a can decrease significantly in the controlled decompression group. Controlled decompression technology maybe decreases the release of TNF-a by regulating the pathway of necroptosis and apoptosis. On the other hand, TNF-a can activate the pathway of necroptosis and apoptosis. Li et al. also reported that necroptosis plays a vital role in regulating its pro-death kinase activity in response to TNF- $\alpha$ and pro-survival activity in response to tolllike receptors (TLRs) signaling ${ }^{33}$. Yun et al. reported that signal transducer and activator of transcription 3 (STAT3) activation in microglia can increase TNF- $\alpha$ expression, then it can increase neuronal apoptosis by increasing reactive oxygen species (ROS) levels in the neuronal cells ${ }^{34}$.

In this study, by comparing the expression of necroptosis markers under three treatment modes, the expression of RIP1 and RIP3 was found to be significantly reduced under the controlled decompression mode. Therefore, it was concluded that controlled decompression technology can alleviate ischemia-reperfusion injury by regulating the necroptosis pathway. The apoptosis pathway was also evaluated by assessing the expression level of caspase-3, which was activated and overexpressed in the TBI model. The caspase-3 inhibitor can reduce the apoptosis of neurons ${ }^{35}$. Mazumder et al. indicated that caspase- 3 is an important factor in the apoptosis process ${ }^{36}$. This study 
showed that caspase- 3 in the controlled decompression group was significantly decreased compared with the rapid decompression group. Therefore, a controlled decompression technique can confer neuroprotection after intracranial hypertension injury via regulated necroptosis and apoptosis.

\section{Conclusion}

Compared with rapid decompression, controlled decompression technology can effectively reduce neuronal death after craniocerebral injury, alleviate ischemiareperfusion injury, and consequently protect brain tissue. Controlled decompression is a new surgical method based on conventional rapid decompression that should be translated into use. The mechanism for minimizing intraoperative and postoperative decompression complications might be by inhibiting the pathway of necroptosis to a certain extent after craniocerebral injury. However, the precise mechanism should be established.

\section{Authors' contribution}

Design of the study: Zhang C, Chen J, Yang $S$ and Wang Y; Critical revision: Zhang C, Chen J, Wang Y; Technical procedures: Wang $Y$, Yang $S$; Acquisition of data: Zhang $\mathrm{C}$ and Chen J; Manuscript writing: Zhang $\mathrm{C}$, Wang $\mathrm{Y}$ and Chen J; Final approval: Zhang C, Chen J, Yang S and Wang Y.

\section{Data availability statement}

Data will be available upon request.

\section{Funding}

Wuxi Health and Family Planning Bureau

Grant No. Z01705

Wuxi Key Disciplines

Grant No. ZDXK005

\section{Acknowledgments}

Not applicable.

\section{References}

1. Gao G, Wu X, Feng J, Hui J, Mao Q, Lecky F, et al. Clinical characteristics and outcomes in patients with traumatic brain injury in China: a prospective, multicentre, longitudinal, observational study. Lancet Neurol. 2020;19(8):670-7. https://doi.org/10.1016/S1474-4422(20)30182-4
2. Corrigan J, Selassie A, Orman JA. The Epidemiology of Traumatic Brain Injury.J Head Trauma Rehabil. 2010;25(2):7280. https://doi.org/10.1097/HTR.0b013e3181ccc8b4

3. Kienzler J, Zakelis R, Bäbler S, Remonda E, Ragauskas A, Fandino J. Validation of Noninvasive Absolute Intracranial Pressure Measurements in Traumatic Brain Injury and Intracranial Hemorrhage. Oper Neurosurg (Hagerstown). 2019;16(2):186-96. https://doi.org/10.1093/ons/opy088

4. Chen J-H, Li P-P, Yang L-K, Chen L, Zhu J, Hu X, et al. Value of Ventricular Intracranial Pressure Monitoring for Traumatic Bifrontal Contusions. World Neurosurg. 2018;113:e690e701. https://doi.org/10.1016/j.wneu.2018.02.122

5. BrownDA,WijdicksEFM.Chapter16-Decompressivecraniectomy in acute brain injury. Handb Clin Neurol. 2017;140:299-318. https://doi.org/10.1016/B978-0-444-63600-3.00016-7

6. Stein SC, Georgoff P, Meghan S, Mirza KL, El Falaky OM. Relationship of aggressive monitoring and treatment to improved outcomes in severe traumatic brain injury. J Neurosurg. 2010;112(5):1105-12. https://doi. org/10.3171/2009.8.JNS09738

7. Mcleod A. Traumatic injuries to the head and spine 1: mechanisms of injury. Br J Nurs. 2004;13(16):940-7. https://doi.org/10.12968/bjon.2004.13.16.15963

8. Wang Y, Wang C, Yang L, Cai S, Cai X, Dong J, et al. Controlled Decompression for the Treatment of Severe Head Injury: A Preliminary Study. Turk Neurosurg. 2014;24(2):214-20. https://doi.org/10.5137/1019-5149.JTN.8135-13.1

9. Chen J, Li M, Chen L, Chen W, Zhang C, Feng Y, et al. The Effect of Controlled Decompression for Severe Traumatic Brain Injury: A Randomized, Controlled Trial. Front Neurol. 2020;11:107. https://doi.org/10.3389/fneur.2020.00107

10. Moriwaki K, Chan FK-M. Chapter Seven - The Inflammatory Signal Adaptor RIPK3: Functions Beyond Necroptosis. Int Rev Cell Mol Biol. 2017;328:253-75. https://doi. org/10.1016/bs.ircmb.2016.08.007

11. Degterev A, Huang Z, Boyce M, Li Y, Jagtap P, Mizushima $\mathrm{N}$, et al. Chemical inhibitor of nonapoptotic cell death with therapeutic potential for ischemic brain injury. Nat Chem Biol. 2005;1:112-9. https://doi.org/10.1038/nchembio711

12. Vasilikos L, Spilgies LM, Knop J, Wong WW-L. Regulating the balance between necroptosis, apoptosis and inflammation by inhibitors of apoptosis proteins. Immunol Cell Biol. 2017;95(2):160-5. https://doi.org/10.1038/icb.2016.118

13. Shan B, Pan H, Najafov A, Yuan J. Necroptosis in development and diseases. Genes Dev. 2018;32:327-40. https://doi.org/10.1101/gad.312561.118

14. Ito Y, Ofengeim D, Najafov A, Das S, Saberi S, Li Y, et al. RIPK1 mediates axonal degeneration by promoting inflammation and necroptosis in ALS. Science. 2016;353(6299):603-8. https://doi.org/10.1126/science.aaf6803

15. Ke X, Lei L, Li H, Li H, Yan F. Manipulation of necroptosis by Porphyromonas gingivalis in periodontitis development. 
Mol Immunol. 2016;77:8-13. https://doi.org/10.1016/j. molimm.2016.07.010

16. Guan H, Zhang C, Chen T, Zhu J, Yang S, Shu L, et al. Controlled Decompression Attenuates Brain Injury in a Novel Rabbit Model of Acute Intracranial Hypertension. Med Sci Monit. 2019;25:9776-85. https://doi.org/10.12659/MSM.919796

17. Chen J-H, Yang L-K, Chen L, Wang $\mathrm{Y}-\mathrm{H}$, Wu Y, Jiang B-J, et al. Atorvastatin ameliorates early brain injury after subarachnoid hemorrhage via inhibition of AQP4 expression in rabbits. Int J Mol Med. 2016;37:1059-66. https://doi.org/10.3892/ijmm.2016.2506

18. Chen J-H, Wu T, Yang L-K, Chen L, Zhu J, Li P-P, et al. Protective effects of atorvastatin on cerebral vessel autoregulation in an experimental rabbit model of subarachnoid hemorrhage. Mol Med Rep. 2018;17:1651-9. https://doi. org/10.3892/mmr.2017.8074

19. Chen J-H, Wu T, Xia W-Y, Shi Z-H, Zhang C-L, Chen L, et al. An early neuroprotective effect of atorvastatin against subarachnoid hemorrhage. Neural Regen Res. 2020;15(10):1947-54. https://doi.org/10.4103/16735374.280326

20. Chen J, Xuan $Y$, Chen $Y$, Wu T, Chen L, Guan $H$, et al. Netrin-1 alleviates subarachnoid haemorrhage-induced brain injury via the PPARY/NF-KB signalling pathway. J Cell Mol Med. 2019;23(3):2256-62. https://doi.org/10.1111/ jcmm.14105

21. Chen $Y$, Wu $X, Y u S$, Fauzee NJS, Wu J, Li L, et al. Neuroprotective Capabilities of Tanshinone IIA against Cerebral Ischemia/Reperfusion Injury via Anti-apoptotic Pathway in Rats. Biol Pharm Bull. 2012;35(2):164-70. https://doi.org/10.1248/bpb.35.164

22. Willis EF, MacDonald KPA, Nguyen QH, Garrido AL, Gillespie ER, Harley SBR, et al. Repopulating Microglia Promote Brain Repair in an IL-6-Dependent Manner. Cell. 2020;180(5):83346. https://doi.org/10.1016/j.cell.2020.02.013

23. Shiban $E$, Lange $N$, Hauser A, Jörger A-K, Wagner A, Meyer $B$, et al. Cranioplasty following decompressive craniectomy: minor surgical complexity but still high periprocedural complication rates. Neurosurg Rev. 2020;43:217-22. https://doi.org/10.1007/s10143-018-1038-x

24. Su T-M, Lee T-H, Chen W-F, Lee T-C, Cheng C-H. Contralateral Acute Epidural Hematoma After Decompressive Surgery of Acute Subdural Hematoma: Clinical Features and Outcome. J Trauma. 2008;65(6):1298-302. https://doi.org/10.1097/ TA.0b013e31815885d9

25. Jiang X, Ai C, Shi E, Nakajima Y, Ma H. Neuroprotection against Spinal Cord Ischemia-Reperfusion Injury Induced by Different Ischemic Postconditioning Methods: Roles of Phosphatidylinositol 3-Kinase-Akt and Extracellular Signalregulated Kinase. Anesthesiology. 2009;111:1197-205. https://doi.org/10.1097/ALN.0b013e3181bf1d93
26. Tamaki $T$, Node $\mathrm{Y}$, Yamamoto $\mathrm{Y}$, Teramoto $A$. Cardiopulmonary Hemodynamic Changes During Acute Subdural Hematoma Evacuation. Neurol Med Chir (Tokyo). 2006;46(5):219-25. https://doi.org/10.2176/nmc.46.219

27. Chen J, Wang Y, Wu J, Yang J, Li M, Chen Q. The Potential Value of Targeting Ferroptosis in Early Brain Injury After Acute CNS Disease. Front Mol Neurosci. 2020;13:110. https://doi.org/10.3389/fnmol.2020.00110

28. Newton K, Dugger DL, Wickliffe KE, Kapoor N, Almagro MC, Vucic D, et al. Activity of Protein Kinase RIPK3 Determines Whether Cells Die by Necroptosis or Apoptosis. Science. 2014;343(6177):1357-60. https://doi.org/10.1126/ science.1249361

29. Weinlich R, Oberst A, Beere HM, Green DR. Necroptosis in development, inflammation and disease. Nat Rev Mol Cell Biol. 2017;18:127-36. https://doi.org/10.1038/ nrm.2016.149

30. Yamanaka K, Saito Y, Yamamori T, Urano Y, Noguchi N. 24(S)Hydroxycholesterol Induces Neuronal Cell Death through Necroptosis, a Form of Programmed Necrosis. J Biol Chem. 2011;286(28):24666-73. https://doi.org/10.1074/jbc. M111.236273

31. Liu T, Zhao D-x, Cui H, Chen L, Bao Y-h, Wang $\mathrm{Y}$, et al. Therapeutic hypothermia attenuates tissue damage and cytokine expression after traumatic brain injury by inhibiting necroptosis in the rat. Sci Rep. 2016;6:24547. https://doi.org/10.1038/srep24547

32. Yang J, Zhao Y, Zhang L, Fan H, Qi C, Zhang K, et al. RIPK3/ MLKL-Mediated Neuronal Necroptosis Modulates the M1/ M2 Polarization of Microglia/Macrophages in the Ischemic Cortex. Cereb Cortex. 2018;28(7):2622-35. https://doi. org/10.1093/cercor/bhy089

33. Li X, Zhang $M$, Huang $X$, Liang $W$, Li G, Lu X, et al. Ubiquitination of RIPK1 regulates its activation mediated by TNFR1 and TLRs signaling in distinct manners. Nat Commun. 2020;11:6364. https://doi.org/10.1038/s41467020-19935-y

34. Yun J-H, Lee D-H, Jeong H-S, Kim HS, Ye S-K, Cho C-H. STAT3 activation in microglia exacerbates hippocampal neuronal apoptosis in diabetic brains. J Cell Physiol. 2021. https:// doi.org/10.1002/jcp.30373

35. Springer JE, Nottingham SA, McEwen ML, Azbill RD, Jin Y. Caspase-3 Apoptotic Signaling Following Injury to the Central Nervous System. Clin Chem Lab Med. 2001;39(4):299-307. https://doi.org/10.1515/CCLM.2001.046

36. MazumderL S, Plesca D, Almasan A. Caspase-3 Activation is a Critical Determinant of Genotoxic Stress-Induced Apoptosis. In: Mor G, Alvero AB, editors. Apoptosis and Cancer. Methods in Molecular Biology. Totowa: Humana; 2008. p.13-21. https://doi.org/10.1007/978-1-59745-3394_2 